\title{
Exploring Cascading Outages and Weather via Processing Historic Data
}

\author{
Ian Dobson Nichelle'Le K. Carrington \\ Kai Zhou Zhaoyu Wang \\ ECpE Department, Iowa State University \\ Ames Iowa USA dobson@iastate.edu
}

\author{
Benjamin A. Carreras \\ BACV Solutions \\ Oak Ridge TN USA \\ bacarreras@gmail.com
}

\author{
José M. Reynolds-Barredo \\ Departamento de Física \\ Universidad Carlos III Madrid, Spain \\ jmrb2002@gmail.com
}

\begin{abstract}
We describe some bulk statistics of historical initial line outages and the implications for forming contingency lists and understanding which initial outages are likely to lead to further cascading. We use historical outage data to estimate the effect of weather on cascading via cause codes and via NOAA storm data. Bad weather significantly increases outage rates and interacts with cascading effects, and should be accounted for in cascading models and simulations. We suggest how weather effects can be incorporated into the OPA cascading simulation and validated. There are very good prospects for improving data processing and models for the bulk statistics of historical outage data so that cascading can be better understood and quantified.
\end{abstract}

\section{Introduction}

Cascading failure can be defined as a sequence of dependent outages that successively weakens or degrades the power transmission system [1]. Although the power transmission system is carefully designed and operated to be robust to multiple outages, cascading outages that are large enough to cause load shedding and blackouts do occur. The large cascading blackouts that are of the greatest concern are infrequent, but likely enough to have substantial risk [2-5].

Cascading is the general way that transmission blackouts become widespread and there are many mechanisms that contribute to initial outages or the subsequent propagation of outages. There are a correspondingly large variety of models, approximations, simulations, and procedures to assess and mitigate cascading outages $[1,6]$. One way to evaluate and improve these efforts is validation with observed historical data [7-9]. There is now much more systematic and automated collection of outage data by utilities, but the challenges of extracting and processing useful information from the data remain.
In this paper, we report on some bulk statistical processing of 14 years of transmission line outage data from a large North American utility to describe initial line outages and to start to explore the effect of weather on cascading. Our data-driven analysis of the effect of weather on the bulk statistics of cascading and aspects of our bulk statistical analysis of initial line outages are novel. Incorporating some of these effects in the OPA (Oakridge-Pserc-Alaska) cascading blackout simulation is also considered (see summary of OPA in section 7).

Instead of working directly with data as in this paper, one can make simulation models that use or are tuned to typical parameter values. Several authors have taken this approach to propose models of weather effects in cascading simulations [10-13].

While historical data processing has many advantages, including no modeling assumptions and a very favorable grounding in reality, it should be noted that the grid evolves over 14 years, and that statistical analysis of historical cascades necessarily describes cascading risk averaged over the time period of observation.

\section{Historical outage data and its processing}

The transmission line outage data consists of 42561 automatic and planned line outages recorded by a North American utility over a period of 14 years starting in January 1999 [14]. The data includes the outage start time (to the nearest minute), names of the buses at both ends of the line, and the dispatcher cause code. The automatic line outages are identified. All this data is standard and routinely collected by utilities. For example, this data is reported by North American utilities in NERC's Transmission Availability Data System (TADS) $[15,16]$ and is also collected in other countries.

Having formed the network model from the automatic and planned line outages [17], the analysis of cascading focuses on only the automatic outages. There are 10942 automatic outages in the data. The network model has 614 lines, 361 buses and is a connected network. 
The structure of cascading is that each cascade starts with initial outages in the first generation followed by further outages grouped into subsequent generations until the cascade stops [18]. The first step in processing the line outages is to group the line outages into individual cascades, and then within each cascade to group the outages that occur in close succession into generations. The grouping of the outages into cascades and generations within each cascade is done based on the outage start times according to the methods of $[19,20]$. We summarize the procedure here and refer to [20] for the details. The grouping is done by looking at the gaps in start time between successive outages.

If successive outages have a gap of one hour or more, then the outage after the gap starts a new cascade. (That is, suppose $o_{1}, o_{2}, \ldots$ are the outage start times in their order of occurrence. A gap of more than one hour is defined as a time interval between successive outage start times $o_{i}, o_{i+1}$ such that $o_{i+1}-o_{i} \geq 1$ hour. The time before the first outage start time $o_{1}$ and the time after the last outage start time are also considered to be gaps of more than hour. Let $g_{1}, g_{2}, \ldots$ be all the gaps of more than one hour in their order of occurrence. Then cascade number $k$ is defined to be all the outages that have start times between the gaps $g_{k}$ and $g_{k+1}$. An alternative and equivalent definition is that a cascade is a maximal series of outages with successive outage start times for which the time difference between successive outage start times in the series is less than one hour. A cascade may consist of one outage or many outages.)

Within each cascade, if successive outages have a gap of more than one minute, then the outage after the gap starts a new generation of the cascade. (That is, suppose $o_{k, 1}, o_{k, 2}, \ldots$ are the outage start times in their order of occurrence for all the outages in cascade $k$. A gap of more than one minute is defined as a time interval between successive outage start times $o_{k, i}, o_{k, i+1}$ such that $o_{k, i+1}-o_{k, i}>1$ minute. The time before the first outage start time $o_{k, 1}$ in the cascade $k$ and the time after the last outage start time in cascade $k$ are also considered to be gaps of more than minute. Let $g_{k, 1}, g_{k, 2}, \ldots$ be all the gaps of more than one minute in cascade $k$ in their order of occurrence. Then generation number $\ell$ of cascade number $k$ is defined to be all the outages in cascade $k$ that have start times between the gaps $g_{k, \ell}$ and $g_{k, \ell+1}$.) Since the outage times are only known to the nearest minute, the order of outages within a generation often cannot be determined.

This simple method of defining cascades and generations of outages appears to effective and has gap thresholds consistent with power system time scales since operator actions are usually completed within one hour and fast transients and protection actions such as auto- reclosing are completed within one minute. [20] examines the robustness of cascade propagation with respect to varying these gap thresholds.

This data processing applied to the 10942 automatic outages yields 6687 cascades. Most of the cascades are short: $84 \%$ of the cascades have only the first generation of outages and do not spread beyond these initial outages. It is important for a fair statistical analysis to include the short cascades (even if they are for other purposes not thought of as cascades); the short cascades usually represent a successful case of resilience in which no load is shed. That is, excluding the short cascades would misleadingly bias the results towards the more damaging cascades that do not stop quickly.

The grouping of outages in each cascade into generations allows the initial outages in the first generation to be distinguished from the subsequently cascading outages in the following generations. This is of interest because the mechanisms and mitigations of the initial line outages differ significantly from the interactions between line outages that are involved in the subsequent cascade.

Most of the initial line outages are single outages, but there are also multiple initial outages. In other words, there are single, double, triple, etc. contingencies. The probability distribution of the number of initial outages is shown by the black dots in Figure 1. The distribution of the initial outages is one way of looking at the severity of initial events: $12 \%$ of the initial events have more than one outage (the probability of one initial outage is $88 \%$ ), $1.5 \%$ of the initial events have more than 3 outages, and $0.2 \%$ have more than 5 outages.

Cascading increases the probabilities of multiple line outages. The distribution of the total number of outages after cascading is shown by the red squares in Figure $1.26 \%$ of the total number of outages have more than one outage, $6.6 \%$ of the total number of outages have more than 3 outages, $2.7 \%$ have more than 5 outages, and $0.7 \%$ have 10 or more outages. The effect of cascading is progressively larger for the cascades with more outages. For example, while cascading approximately doubles the probability of more than one outage, cascading increases the probability of more than 5 outages by an order of magnitude.

The generations of outages in the cascades are analogous to human generations; parents in one generation give rise to children in the next generation. The average propagation per parent $\lambda$ is the total number of child outages in all the generations divided by the total number of parent outages in all the generations. $\lambda$ calculated from our data is 0.28 . That is, each outage in a generation will, on average, be followed by 0.28 outages in the next generation. $\lambda$ quantifies in an overall way how much 


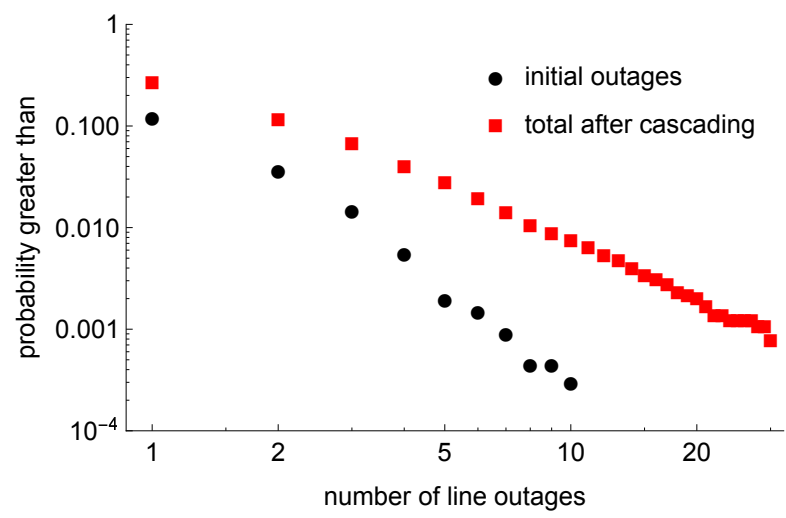

Figure 1. Probability of more than $k$ outages vs. $k$ for the initial line outages and for the total line outages after cascading

cascading increases the number of line outages starting from the initial line outages.

\section{Statistics of initial outages}

We examine the basic statistics of the automatic initial line outages (those outages in the first generation of cascading). The annual outage frequencies $\mu_{1}, \mu_{2}, \mu_{3}, \ldots, \mu_{614}$ for the 614 lines range from zero outages to 23 outages per year, with a mean annual frequency $\bar{\mu}=0.92$ outages per year.

The large variation in initial line outage frequency $\mu_{i}$ in this data has several implications. It is clear that cascading simulations that aim to quantify cascading risk should sample from realistic initial line outage frequencies. One way to accomplish this is to simulate a real power system and use the observed historical frequency of line outages. Another way to accomplish this is to understand and model the factors or characteristics that largely determine the frequency of initial line outages so that they can be represented in artificial power system models. For example, it might be expected that outage frequency has some dependence on line length and other characteristics. (Our data suggests a mild correlation of 0.3 between outage frequency and line length for lines between 1 and 50 miles long.) Another implication is that it may be difficult to classify the probability of higher order initial outages (when assumed roughly independent) by the number of outages using the order of magnitude of the probabilities [21] because the single outages vary so much in their probability.

The data in Figure 1 shows a substantial probability of multiple initial outages. The empirical probability of two initial outages is 0.084 with standard deviation 0.0034 . To determine whether this can arise from independent single line outages, we suppose that each of the
614 lines has initial outages according to a Poisson process of rate $368 / 614=0.60$ outages per year and that the Poisson processes for different lines are independent. Then the outages of any of the lines is a Poisson process of rate 368 outages per year, which matches the rate extracted from data in the next paragraph. Multiple initial line outages in the data require at least 2 line outages to occur at times that are either in the same or adjacent minutes when the times are quantized to minutes. Given the first outage time, this requires the second outage to occur within a 3 minute interval. (For example, if the quantization works by quantizing the time $t$ in minutes to $\lfloor t\rfloor$, the greatest integer number of minutes less than $t$, then $t_{1}$ and $t_{2}$ are in the same or adjacent quantized minutes if and only if $\left\lfloor t_{1}\right\rfloor-1<t_{2} \leq\left\lfloor t_{1}\right\rfloor+2$.) The probability that the second outage occurs within a given 3 minute interval is $1-\exp [-(3 \times 368) / 525600]=0.002$. Therefore the probability of multiple initial outages and in particular the probability of 2 initial outages are both bounded above by 0.002 . Since the empirical probability of 2 initial outages is 0.084 , an order of magnitude greater than 0.002 , the double initial outages are dependent and cannot be regarded as mainly arising from independent single line outages. It also follows that multiple initial line outages are dependent. A similar claim of dependence for all outages (not just the initial outages) is established in previous work [20-22].

The previous paragraph assumes that initial outages are a Poisson process of rate 368 outages per year. The assumption that initial outages are a Poisson process is supported by examining the distribution of the logarithm of time differences between successive outage times in Figure 2. For an exact Poisson process, the time differences follow an exponential distribution with the same rate as the Poisson process, so that the logarithm of the survival function of the time differences is a linear function with the slope of the line determining the rate. The time differences of both the initial outages and all the outages are approximately linear except for the smaller time differences of order one hour or less. (The increased, superlinear number of smaller time differences in all the outages may be attributed to cascading. There are no time differences between initial outages more than one minute and less than one hour because of the data processing that defines the start of new cascades. The initial and all outages also show a fraction of outages that have a time difference less than one minute or zero.) The dotted line in Figure 2 approximates the slope of the time differences except for the smaller time differences of order one hour or less and has a slope corresponding to 368 outages per year. The Poisson process model for initial outages is similar to the Poisson process model of blackout start times analyzed in [5], except that 


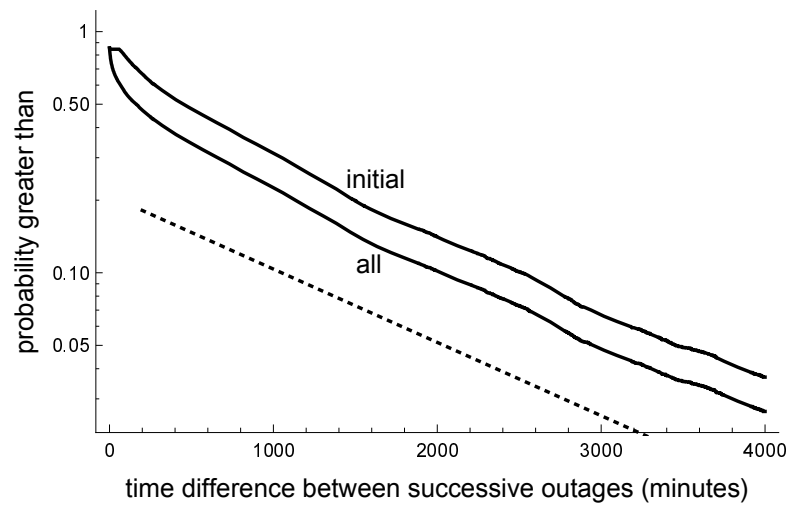

Figure 2. Probability distribution (survival function) of time differences between successive initial line outages (upper curve) and all line outages (lower curve). Note the logarithmic vertical scale. Dotted straight line has slope corresponding to an exponential distribution of time differences with rate 368 outages per year.

\section{Table 1. Double initial line outages by type}

\begin{tabular}{ccccc} 
outaged lines & all & weather & no weather & random \\
\hline adjacent & $55 \%$ & $53 \%$ & $55 \%$ & $2 \%$ \\
parallel & $20 \%$ & $11 \%$ & $24 \%$ & $0 \%$ \\
separated & $16 \%$ & $26 \%$ & $11 \%$ & $97 \%$ \\
repeated & $9 \%$ & $10 \%$ & $9 \%$ & $1 \%$
\end{tabular}

here we do not consider any adjustment to the Poisson process to account for a slowly varying rate.

The multiple initial outages have significant spatial dependence. Consider the classification of initial double outages in Table 1. The double outages are either two adjacent lines (lines with exactly one bus in common), two parallel lines (two buses in common), two separated lines (no common buses), or are repeat outages of the same line. More than half (55\%) of the double outages are adjacent lines and only $16 \%$ of the double outages are two lines that are separated in the network. In contrast, randomly sampling double line outages by choosing each of the double lines randomly proportional to their outage frequency yields only $2 \%$ that are two adjacent lines and 97\% that are two lines that are separated in the network. Combining the adjacent, parallel, and repeated outages shows that $84 \%$ of the initial double line outages form connected subgraphs.

Outages can be caused by line, bus or breaker faults. Line faults are isolated by the breakers at each end of the line so that they usually cause single line outages, while the bus or breaker faults can cause multiple outages because of the substation protection system design. Although we do not know any specifics of the substation designs, one likely cause of the high proportion of adjacent double line initial outages is substation breaker schemes that disconnect two lines for certain faults.

$2 \%$ of the initial outages are triple outages and $75 \%$ of these initial triple outages are connected subgraphs. $4 \%$ of the initial outages have 3 or more outages and $68 \%$ of these initial multiple outages are connected subgraphs. Spatially close components that are assumed to always outage together for cascading failure analysis are called protection control groups [23] or functional groups [21]. While there clearly would be some overlap, we do not yet know how exactly the connected subgraphs that form the majority of these initial outages are related to the functional groups that can be applied to approximate the protection system actions. The historical data samples from functional groups, but also samples from rarer or more unusual conditions.

It can also be helpful for risk analysis to find out whether different types of multiple initial outages can cause subsequent outages. Our data shows that separated initial line outages are more likely to trigger subsequent cascading outages: $25 \%$ of separated initial line outages have subsequent outages, while only $16 \%$ of connected initial line outages have subsequent outages. The Mann-Whitney test shows this difference is significant at the 0.01 level (p-value is 0.00355 ). For momentary initial outages (duration less than one minute) versus non-momentary initial outages, our data shows little difference in the proportion of subsequent cascading outages: $18 \%$ of momentary outages have subsequent outages, and $15 \%$ of non-momentary outages have subsequent outages. The Mann-Whitney test shows that this difference is not significant at the 0.01 level (pvalue is 0.0197 ). This suggests that momentary and nonmomentary initial outages be treated equally in assessing the risk of further cascading.

The distinction in the processed line outage data between initial outages and subsequently cascading outages allows us to find out and compare which lines are most involved in these two different processes. The top 10 lines involved in initial outages overlap, but do not coincide with the top 10 lines involved in subsequent cascading; there are 6 lines in common but there are 4 lines in each list that differ. Similarly, the top 20 lines involved in initial outages have 10 lines in common with the top 20 lines involved in subsequent cascading and 10 lines in each list that differ. Similar results were obtained by processing line outage data from a neighboring utility [9]. It should be noted that statistically prominent outage problems in an initial portion of the 
historical data may have been already mitigated.

\section{Effect of weather and other influences via cause codes}

The dispatcher outage cause codes allow classification of the cascades of outages into two groups: weather related and non-weather related. (For definiteness when the field and dispatcher causes differ, we do not consider the field cause codes in this analysis.) A cascade of outages is defined as weather related when at least one of the outages in the cascade has one of the cause codes "Weather", "Lightning", "Galloping Conductors", "Ice", "Wind", or "Tree blown".

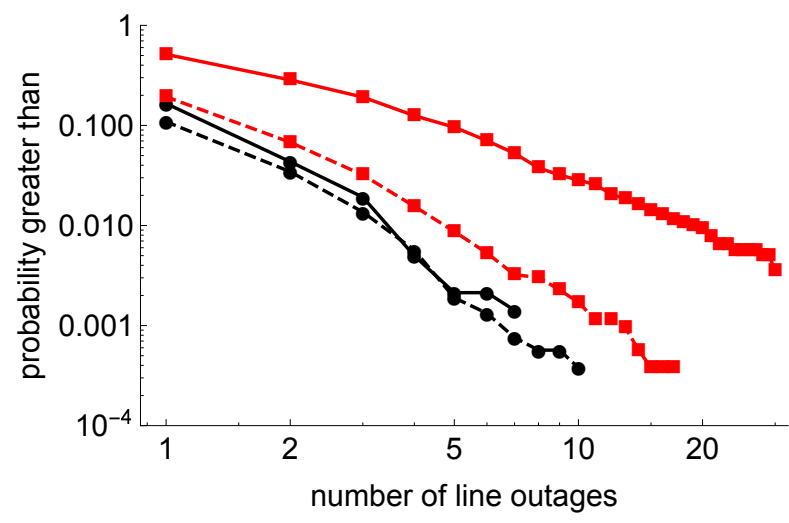

Figure 3. Probability distributions of initial (black circles) and cascaded (red squares) outages with weather (solid line) and no weather (dashed line). Weather is determined by cause code.

How the annual cascade rate, average propagation of cascading outages, and cascade size distribution depend on weather are shown in Table 2 and Figure 3. According to Table 2, only $21 \%$ (101/478) of the cascades are weather related. This implies that only $21 \%$ of the initial outages occurred in a weather-related cascade. And Figure 3 shows that the distribution of initial outages is similar for weather and non-weather related cascades. However, in Table 2 weather related outages have greatly increased propagation from 0.13 (non-weather related) to 0.55 (weather related) and in Figure 3 there is a corresponding large difference in the distribution of the total outages in a cascade after the cascading. That is, a minority of cascades are weather related, but they propagate far more to form larger cascades.

With the method of processing outages into cascades that we use [20], propagation can arise both from outages causing further outages through interactions in the network (encompassing electrical physics, control sys- tems, and human actions) and through independent outages occurring during the cascade that are similar in mechanism to the initial outages. Note that the processing method defining the subsequent cascading studiously avoids determining the causes or explicit dependences of further outages and simply accounts for outages that occur within one hour of previous outages [20]. Indeed [20] states that "One caution is that it is unknown to what extent exogenous forcing from weather is augmented by additional dependent cascading effects." Reference [17] analyzes all the outages together, determines the average rate of independent outages, and proceeds to quantify the contribution of statistically independent outages towards the $\lambda$ measure of average propagation, concluding that $4-6 \%$ of outages are independent and classified as cascading outages. This seems an acceptable error in the contention that the subsequently cascading outages are dependent outages. The contention essentially relies on the independent outages occurring at a slow enough rate relative to the typical cascade duration.

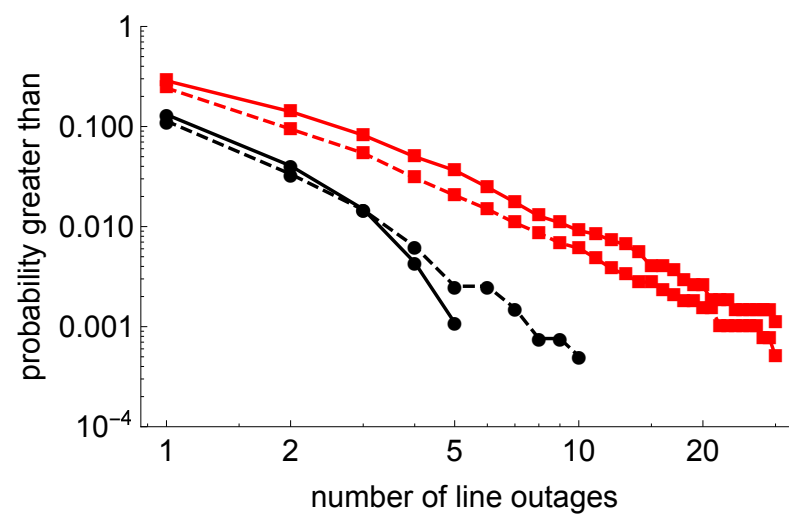

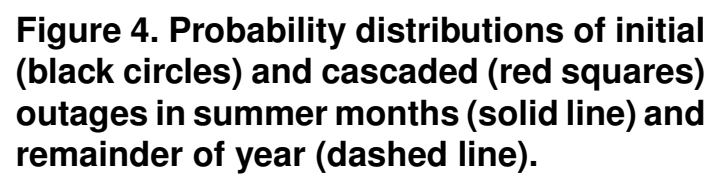

However, the same contention for the subset of weather related outages need not have an acceptable error because there is a much higher rate of independent outages during bad weather. The methods of Section 5 are not conclusive in this regard, but the results of Section 5 are consistent with this conclusion. More importantly, traditional risk analysis does support a much higher rate of independent outages during bad weather $[24,25]$. This raises a question of the validity of the method of cascading processing applied to weather related outages when the cascades and propagation are interpreted as dependent outages occurring through network interactions. However, if the concern is simply the number of subsequent outages during a one hour period 
Table 2. Some general dependencies of initial outages and average propagation

equivalent annual propagation $N=$ number of outages in cascade

\begin{tabular}{cccccl} 
cascade rate & $\lambda$ & $P[N>1]$ & $P[N>5]$ & $P[N>10]$ & \multicolumn{1}{c}{ CAUSE } \\
\hline 478 & 0.28 & 0.26 & 0.027 & 0.007 & ALL OUTAGES \\
101 & 0.55 & 0.51 & 0.096 & 0.028 & WEATHER \\
377 & 0.13 & 0.19 & 0.009 & 0.002 & NOT WEATHER \\
588 & 0.31 & 0.29 & 0.04 & 0.009 & SUMMER MONTHS \\
423 & 0.25 & 0.24 & 0.02 & 0.006 & NOT SUMMER MONTHS \\
486 & 0.36 & 0.34 & 0.05 & 0.010 & PEAK HOURS \\
475 & 0.25 & 0.24 & 0.02 & 0.007 & NOT PEAK HOURS \\
\hline
\end{tabular}

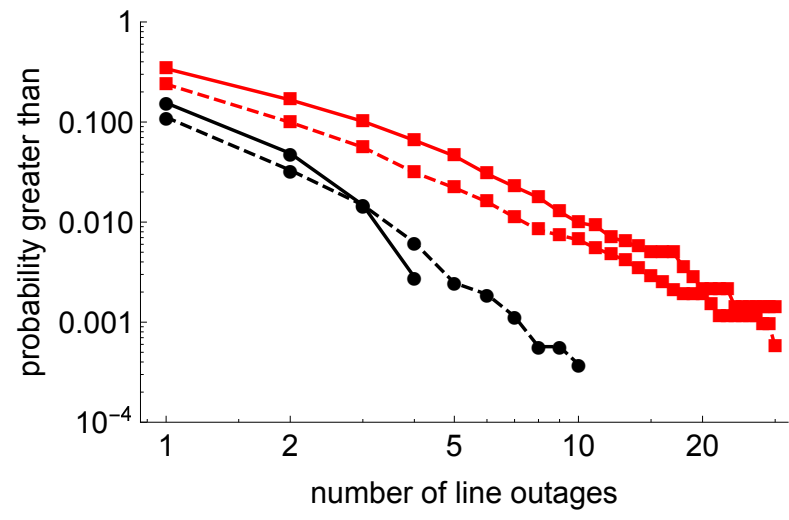

Figure 5. Probability distributions of initial (black circles) and cascaded (red squares) outages at peak hours (solid line) and offpeak hours (dashed line).

without regard to cause, then the method could retain some validity for weather related outages. This is the case, for example, when the concern is the total number of outages, regardless of cause, that the operators have to deal with within a one hour period.

The month and time of day can also be used to classify cascades into the summer peaking months of June, July, August, September, and the remainder of the year, or those cascades that start during the peak hours between $3 \mathrm{pm}$ and $8 \mathrm{pm}$ and cascades starting outside these peak hours. Table 2 and Figures 4 and 5 show the effects of the summer months and the peak hours. (The equivalent annual rate shown in Table 2 is the rate if the condition such as summer months applied all year.) Outages in the summer months of June, July, August, September have a modestly increased propagation from 0.25 (not summer) to 0.31 (summer). Outages in the peak hours between 3 $\mathrm{pm}$ and $8 \mathrm{pm}$ have increased propagation from 0.25 (not peak hours) to 0.36 (peak hours). Note that the cascades also depend on the initial outages. Indeed, the data in the summer months shows a $39 \%$ higher cascade rate and a
$41 \%$ higher rate of initial outages. Overall, there is a moderate increase in cascade propagation during peak hours and only a small increase in propagation, but an increased rate of initial outages in the summer months. However weather effects are larger than either of these factors.

\section{Effect of weather via NOAA storm data}

One problem with analyzing the effect of weather with outage cause codes is that cause codes cannot describe the weather when there is no outage. Therefore the line outage rate during bad weather, a key quantity, cannot be estimated from cause code analysis. Also, the outage cause codes are manually entered, rely on subjective judgment about classifying causes, and in any case include a sizable proportion (22\% of the dispatcher outage cause codes) of causes "Unknown". One way to address these problems with a different bad weather criterion is to coordinate in time and space the outage data with storm weather records.

The National Oceanic and Atmospheric Administration (NOAA) Storm Events Database is a collection of the occurrence of storm events and other significant weather phenomena recorded by NOAA's National Weather Service from 1950 to present [26]. The NOAA historical storm data records for 1999 to 2013 were obtained for analyzing the storm weather effects influencing our outage data. The NOAA storm data includes the event type, event start and end time, and the location within the state by county or zone. The storm event types that we choose to define as a storm are "Blizzard", "Freezing Fog", "Hail", "Heavy Rain", "Heavy Snow", "High Wind", "Ice Storm", "Lightning", "Sleet", "Strong Wind", "Thunderstorm Wind", "Tornado", "Winter Storm", and "Winter Weather".

To associate the line outages with the storm data, we map the buses onto the county they are located in, and describe each zone by the main counties it intersects. A 
line is defined to be in a county if either its sending or receiving end bus is in that county. A line is defined to be in a zone if that zone includes a county that the line is in. This associates each line with a set of counties. In some cases this set of counties only contains one county. A line outage is then classified as a storm outage if it occurs during a storm event in one of the counties in the set of counties associated with that line. It is straightforward to count the number of storm outages of line number $k$ over the period of observation. Also, the total storm durations for a county is the sum of the durations of the storms in that county during the period of observation, and the total storm time for line $k$ over the period of observation is computed as the average over the counties of the total storm durations for the counties that the line is in. Then the line $k$ storm outage rate $R_{\text {line } k}^{\text {storm }}=$ (number of storm outages of line $k$ )/(total storm time for line $k$ ). Finally, the average storm outage rate $R^{\text {storm }}=$ (number of lines $)^{-1} \sum_{k} R_{\text {line } k}^{\text {storm }}$. The non-storm line outage rate and the average non-storm line outage rate $R^{\text {nostorm }}$ are computed similarly.

This data processing approximates the average nonstorm outage rate as $R^{\text {nostorm }}=1.1$ outages per year and the average storm outage rate as $R^{\text {storm }}=8.1$ outages per year. This significant increase in the outage rate during storm weather has important implications for processing historical data and simulating cascading. First of all, models and simulations of cascading should distinguish and separately consider storm weather and non-storm weather periods. This conclusion based on cascading historical data is not surprising given the attention to this distinction in conventional power system reliability [24, 25] and in [10-13]. Secondly, it is also clear from conventional power system reliability that the initial outage rate is higher during bad weather [24,25]. The high outage rate during storms could be primarily due to increases in the initial outage rate alone or to increases in both the initial outage rate and the propagation. The distinction matters to mitigation of cascading because the initial outage mechanisms differ from the mechanisms for the propagation of outages through the network. Thirdly, as already discussed in Section 4, the significant increase of the average storm line outage rate will also impact the processing of cascading outages into generations. It seems that better high-level models that not only distinguish weather and non-weather conditions but also capture and distinguish the rates of independent and dependent outages are needed.

The effect of the storm weather on the distributions of initial and total number of cascaded outages is shown in Figure 6. In comparing Figure 6 to Figure 3, it should be noted that bad weather or its severity is differently defined by the weather cause codes and the storms in
NOAA data.

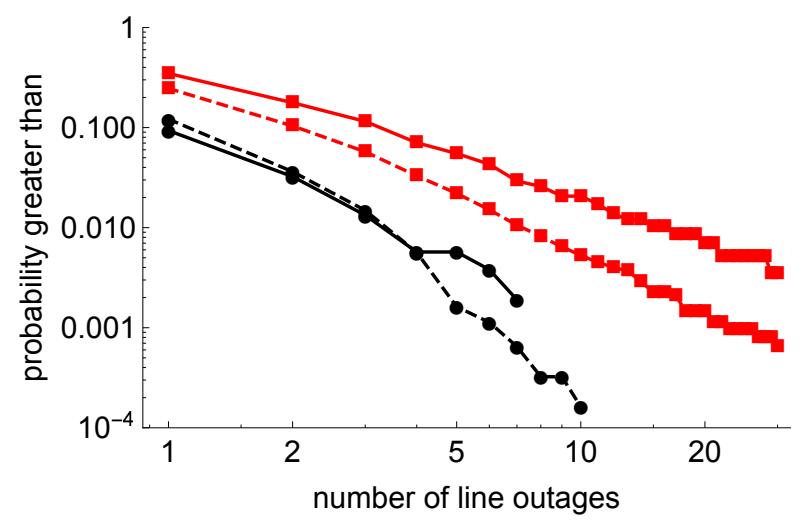

Figure 6. Probability distributions of initial (black circles) and cascaded (red squares) outages with storms (solid line) and no storm (dashed line). Storms are determined by NOAA data.

\section{Cause and effect in cascading analysis}

While an attribution of cause for outages is attractive since it often gives possibilities for mitigation, it should be recognized that the whole notion of detailed cause (and especially single cause) for cascading outages can be murky and ill-defined. Causes for initial outages are less problematic, and often a single cause or multiple causes can be defined. On the other hand, outages propagating via power system interactions after the initial outages generally depend on the initial system condition, the initial outages, and the preceding outages [18].

To suggest an overall methodological context, we can consider two approaches to cascading: A "bottom-up" approach specifies a particular cause and effect mechanism of cascading, makes a detailed model of that mechanism and then tries to get data for that mechanism. A "top-down" approach examines available data, at first without regard to detailed cause or mechanism, and then tries to divide the data into classes of causes or mechanisms. This paper is top-down and weather is one simple example of a class of mechanisms. The bottomup and top-down approaches are complementary. The bottom-up approach enables understanding and often insight into mitigation of that mechanism, but there are dozens of different mechanisms of cascading, and many of the more unusual and complicated events that often occur in blackouts are hard to approach in this way, and very often there is no data available to find the model parameters. The top-down approach already has the data, and can give a useful overall statistical description and 
quantification of cascading, but gives much less detailed insight and as a consequence work towards mitigation is much less direct. However, the operators will have to deal with all the outages in real time regardless of whether there are detailed cause-effect relationships established or not. We hope that bottom-up and top-down approaches will gradually converge towards each other as the field progresses to better realize the full range of possibilities of data-driven reliability analysis.

\section{Modeling weather effects in OPA}

The OPA model [27-29] is a simulation that calculates the long-term risk of cascading blackouts of a power transmission system under the slow, complex dynamics of an increasing power demand and the engineering responses to blackouts. The individual cascades are modeled by probabilistic line overloads and outages in a DC load flow model with linear programming generation redispatch. We previously validated OPA on a 1553 bus model of the Western North American interconnection against observed data with some success [8]. Here we start to explore parameterizing the weather effects in OPA on the 1553 bus model by modifying OPA and showing the fit with observed data. For details of OPA, parameters, and the 1553 bus model we refer to [8].

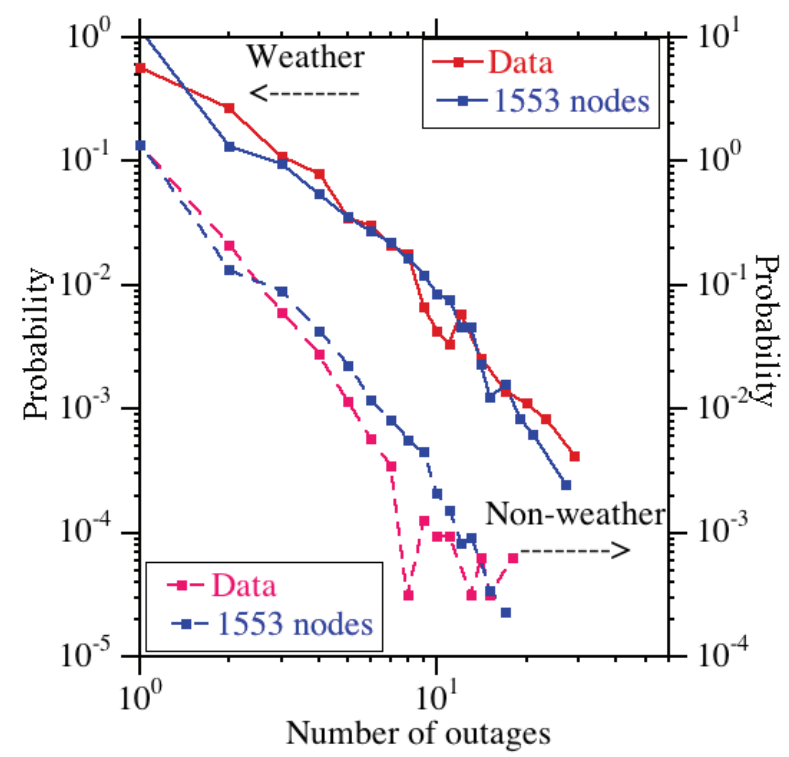

Figure 7. PDFs of number of outages in weather and non-weather cascades from OPA simulation and from observed data.

Inspired by the historical data in the previous sections, we introduce new OPA parameters $p_{w}, p_{5}$ and some spatial correlation between multiple initial outages. $p_{w}$ is the probability that in a given day the weather is the cause of outages. $p_{5}$ is the probability that an outage produced by weather will happen in a given iteration of the cascade on the days that weather is a factor. The spatial correlation of the multiple initial outages is introduced by first using OPA parameter $p_{0}$ to determine some initial line outages. Once initial line outages have been calculated, we go through the adjacent lines and probabilistically determine their failure. Then this process is repeated once. The result is that the initial random line outages are sometimes augmented with one or more adjacent lines.

To reexamine the previous fit with the observed data in [8] with the new parameters, we take $p_{w}=0.25$, which is close to the $21 \%$ proportion of weather cascades estimated in section 4 . We are not yet able to estimate $p_{5}$ from data, but $p_{5}=0.0002$ gives a good match of the cascades with the observed distribution of the number of outages in weather and non-weather cascades as shown in Figure 7. (For $p_{5}<0.0002$ the weather driven cascades tend to be too short, and for $p_{5}>0.0002$ the slope of the weather PDF from the OPA results tends to decrease relative to the slope of the PDF of the data.)

With these parameters, in Figure 8 there is a good match between OPA and the historical data for the distribution of load shed, and in Figure 9 there is a match for the propagation $\lambda$ in each generation of cascading that improves upon the match in [8]. These results suggest that weather effects can be included in OPA and validated against the observed data. Future work should find a way to estimate $p_{5}$ from the historical data instead of calibrating $p_{5}$ with the distribution of the number of outages in weather cascades.

\section{Conclusion}

In this paper we start to explore processing historical outage data to characterize initial outages and subsequent cascading propagation and determine the effects of weather on cascading. Although only one 14 year data set from a large North American utility is analyzed and our specific conclusions are of course limited to that data set, similar data is routinely collected by many utilities worldwide, so that it is straightforward, given access to the data, to apply the data processing methods broadly.

A simple processing method based on outage timing allows us to distinguish the initial outages from the subsequent cascading. Most of these initial outages are single outages that do not have following outages. However, the data also shows significant numbers of multiple initial outages and initial outages that cascade fur- 


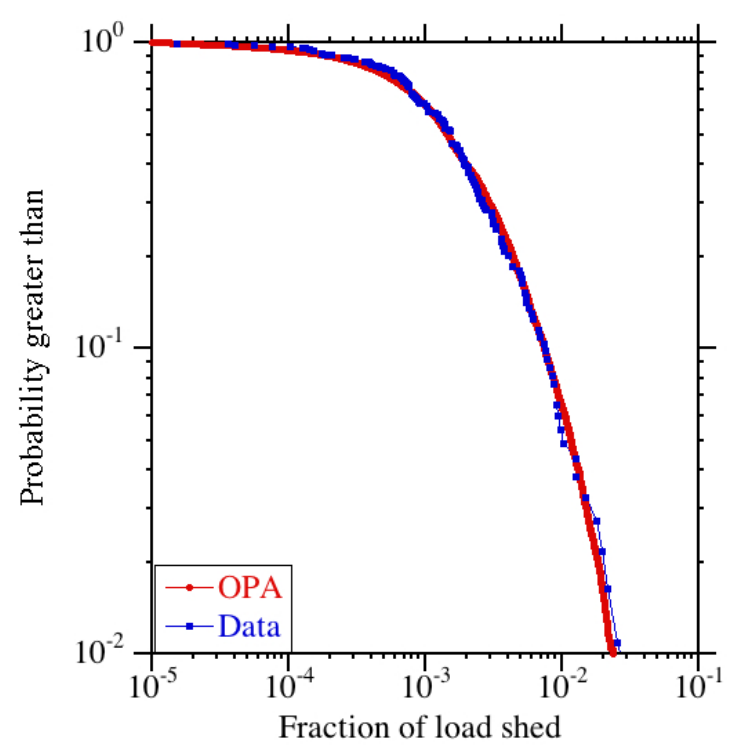

Figure 8. Survival function of fractional load shed for OPA and for Western interconnection historical data from the North American Electric Reliability Corporation.

ther. The initial outages have considerable variation in outage frequency, are dependent, and the multiple outages tend to occur in adjacent lines. However the separated initial multiple outages have more of a tendency to cascade further. Momentary outages appear to cascade further at a similar rate as longer outages. As might be expected from the differing mechanisms involved in initial and propagating outages, the lines most involved in initial outages have some overlap with, but do not coincide with those most involved with subsequent cascading. The bulk statistics of historical initial outages can inform the contingency lists for risk-based or deterministic cascading studies.

The effects of weather on historical cascading outage data are studied by means of the weather-related dispatcher cause codes in outage data and NOAA storm data. A minority of cascades are weather-related, but using the processing methods of the paper, show a significantly increased propagation from the initial outages and a significantly greater outage rate. This suggests that, following traditional power systems risk analysis, cascading models and analysis will need to somehow define and acknowledge bad weather and good weather regimes. An increased outage rate during bad weather is confirmed by traditional power system risk analysis, but its interaction with cascading propagation remains unclear. In particular, the increased outage rate does not allow the increased propagation to be mostly attributed

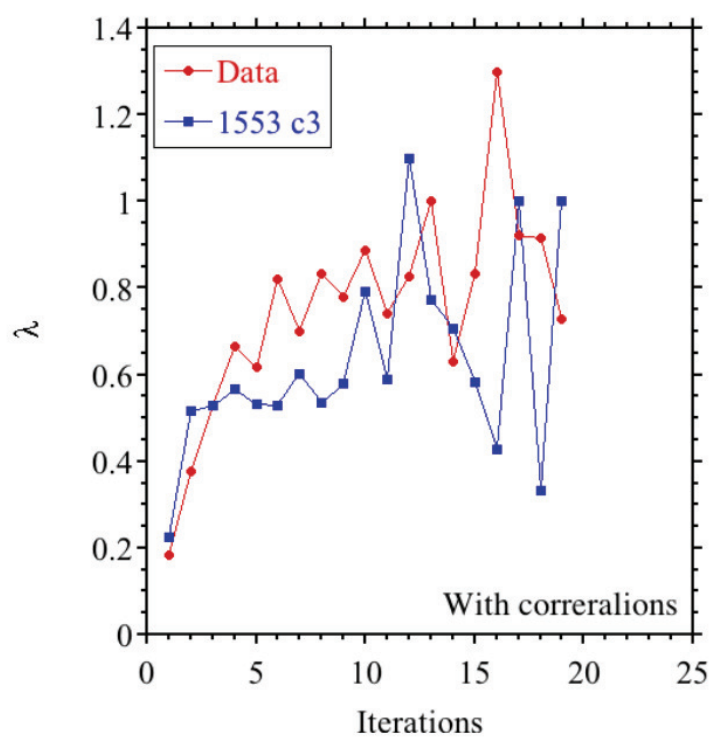

Figure 9. Propagation $\lambda$ at each generation
(iteration) of cascading from OPA and for
the historical data of the paper.

to propagation of cascading via network effects because of limitations of the processing method. New bulk cascading models and data-processing methods are needed for bad weather conditions. Peak hours and peak months of operation show less impact on cascading propagation than bad weather, but there is a higher rate of cascades during these peak conditions.

Historical outage data is very valuable for validating and calibrating simulations of cascading outages. The OPA model of long-term cascading risk is one of the few simulations with some validation with bulk statistics of historical data [8]. We have started to represent weather effects in OPA and extend the validation to this case.

Our bulk statistical data processing methods for historical outage data and NOAA data are initial approaches that are subject to future improvements. However, our results already show the value of this processing for understanding and quantifying key factors in initial outages and subsequent cascading, and the prospects for improved methods and further insights are very good.

Acknowledgements: We gratefully thank Bonneville Power Administration and the National Oceanic and Atmospheric Administration for making publicly available the outage and weather data that made this paper possible. The analysis and any conclusions are strictly those of the authors and not of BPA or NOAA. We gratefully acknowledge support in part from NSF grant 1609080. 


\section{References}

[1] IEEE PES CAMS Task Force on Cascading Failure, Initial review of methods for cascading failure analysis in electric power transmission systems, IEEE PES General Meeting, Pittsburgh PA USA, July 2008.

[2] I. Dobson, B.A. Carreras, V.E. Lynch, D.E. Newman, Complex systems analysis of series of blackouts: cascading failure, critical points, and self-organization, Chaos, vol. 17, no. 2, 026103 June 2007.

[3] P. Hines, J. Apt, S. Talukdar, Large blackouts in North America: historical trends and policy implications, Energy Policy, vol. 37, 2009, pp. 5249-5259.

[4] D.E. Newman, B.A. Carreras, V.E. Lynch, I. Dobson, Exploring complex systems aspects of blackout risk and mitigation, IEEE Trans. Reliability, vol. 60, no. 1, March 2011, pp. 134-143.

[5] B.A. Carreras, D.E. Newman, I. Dobson, North American blackout time series statistics and implications for blackout risk, IEEE Trans. Power Systems, vol. 31, no. 6, November 2016, pp. 4406-4414.

[6] IEEE PES CAMS Task Force on Cascading Failure, Survey of tools for risk assessment of cascading outages, IEEE PES General Meeting, Detroit, MI USA 2011.

[7] IEEE PES CAMS Working Group on Cascading Failure, Benchmarking and validation of cascading failure analysis tools, IEEE Trans. Power Systems, vol. 31, no. 6, November 2016, pp. 4887-4900.

[8] B.A. Carreras, D.E. Newman, I. Dobson, N.S. Degala, Validating OPA with WECC data, 46th Hawaii Intl. Conf. System Sciences, Maui, HI, January 2013.

[9] M. Papic, I. Dobson, Comparing a transmission planning study of cascading with historical line outage data, International Conference on Probability Methods Applied to Power Systems (PMAPS), Beijing China, October 2016.

[10] M.A. Rios et al., Value of security: modeling timedependent phenomena and weather conditions, IEEE Trans. Power Systems, vol. 17, no. 3, 2002, pp. 543-548.

[11] E. Ciapessoni et al., Probabilistic risk-based security assessment of power systems considering incumbent threats and uncertainties, IEEE Trans. Smart Grid, vol. 7, no. 6, Nov. 2016.

[12] F. Cadini, G.L. Agliardi, E. Zio, A modeling and simulation framework for the reliability/availability assessment of a power transmission grid subject to cascading failures under extreme weather conditions, Applied Energy, vol. 185, 2017, pp.267-279.

[13] R. Yao, K. Sun, Towards simulation and risk assessment of weather-related cascading outages, preprint, arXiv:1705.01671 [cs.CE], May 2017.

[14] Bonneville Power Administration Transmission Services Operations \& Reliability website

http://transmission.bpa.gov/Business/Operations/Outages
[15] North American Electric Reliability Corporation (NERC), Transmission Availability Data System (TADS) Data Reporting Instruction Manual, Aug. 2014.

[16] J.J. Bian, S. Ekisheva, A. Slone, Top risks to transmission outages, IEEE PES General Meeting, National Harbor, MD USA, July 2014.

[17] I. Dobson, B.A. Carreras, D.E. Newman, J.M. ReynoldsBarredo, Obtaining statistics of cascading line outages spreading in an electric transmission network from standard utility data, IEEE Trans. Power Systems, vol. 31, no. 6, November 2016, pp. 4831-4841.

[18] I. Dobson, D.E. Newman, Cascading blackout overall structure and some implications for sampling and mitigation, International Journal of Electrical Power Energy Systems, vol. 86, pp. 29-32, 2017.

[19] H. Ren, I. Dobson, Using transmission line outage data to estimate cascading failure propagation in an electric power system, IEEE Trans. Circuits and Systems Part II, 2008, vol. 55, no. 9, pp. 927-931.

[20] I. Dobson, Estimating the propagation and extent of cascading line outages from utility data with a branching process, IEEE Trans. Power Systems, vol. 27, no. 4, November 2012, pp. 2146-2155.

[21] Q. Chen, J.D. McCalley, Identifying high risk N-k contingencies for online security assessment, IEEE Trans. Power Systems, vol. 20, no. 2, May 2005, pp. 823-834.

[22] Q. Chen, C. Jiang, W. Qiu, J.D. McCalley, IEEE Trans. Power Systems, vol.21, no.3, Aug.2006, pp.1423-1431.

[23] R.C. Hardiman, M.T. Kumbale, Y.V. Makarov, An advanced tool for analyzing multiple cascading failures, 8th Intl. Conf. Probability Methods Applied to Power Systems, Ames, Iowa USA, September 2004.

[24] R.Billinton,G.Singh, Application of adverse and extreme adverse weather: Modeling in transmission and distribution system reliability evaluation, IEE Proc.- Gener. Transm. Distrib., vol. 153, no. 1, pp. 115-120, Jan. 2006.

[25] R. Billinton, R. N. Allan, Reliability Evaluation of Power Systems, 2nd ed. New York: Plenum, 1996.

[26] NOAA National centers for environmental information Storm Events Database https://www.ncdc.noaa.gov/stormevents/

[27] I. Dobson, B.A. Carreras, V. Lynch, D.E. Newman, An initial model for complex dynamics in electric power system blackouts, 34th Hawaii International Conference on System Sciences (HICSS), Maui, Hawaii, January 2001.

[28] B.A. Carreras, V.E. Lynch, I. Dobson, D.E. Newman, Complex dynamics of blackouts in power transmission systems, Chaos, vol. 14, no. 3, Sept. 2004, pp. 643-652.

[29] H. Ren, I. Dobson, B.A. Carreras, Long-term effect of the $\mathrm{n}-1$ criterion on cascading line outages in an evolving power transmission grid, IEEE Trans. Power Systems, vol. 23, no. 3, Aug. 2008, pp. 1217-1225. 